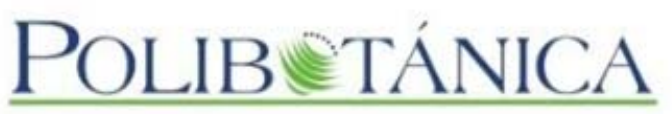

\title{
Polibotánica
}

ISSN electrónico: 2395-9525

polibotanica@gmail.com

Instituto Politécnico Nacional

México

http:www.polibotanica.mx

\section{ASPECTOS TAXONÓMICOS DE LA MORFOLOGÍA FLORAL MASCULINA DE Quercus (FAGACEAE) EN MÉXICO}

\section{TAXONOMIC ASPECTS OF THE MALE FLORAL MORPHOLOGY OF Quercus (FAGACEAE) IN MEXICO}

Romero-Rangel, S., y E. C. Rojas-Zenteno.

ASPECTOS TAXONÓMICOS DE LA MORFOLOGÍA FLORAL MASCULINA DE Quercus (FAGACEAE) EN MÉXICO.

TAXONOMIC ASPECTS OF THE MALE FLORAL MORPHOLOGY OF Quercus (FAGACEAE) IN MEXICO.

POLIBETANICA Instituto Politécnico Nacional
Núm. 48: 13-27 México. Julio 2019

DOI: $10.18387 /$ polibotanica. 48.2

(c) (i) (2) Estete es un artículo de acceso abierto bajo la licencia Creative Commons 4.0 Atribución-No Comercial (CC BY-NC 4.0 Internacional). 


\section{ASPECTOS TAXONÓMICOS DE LA MORFOLOGÍA FLORAL MASCULINA DE Quercus (FAGACEAE) EN MÉXICO}

\section{TAXONOMIC ASPECTS OF THE MALE FLORAL MORPHOLOGY OF Quercus (FAGACEAE) IN MEXICO}

\author{
S. Romero-Rangel / sromero@ unam.mx
E. C. Rojas-Zenteno \\ S. Romero-Rangel / sromero@ unam.mx
E. C. Rojas-Zenteno \\ Laboratorio de Ecología y Taxonomía de Árboles y Arbustos \\ Facultad de Estudios Superiores Iztacala-UNAM \\ Tlalnepantla, Estado de México.
}

Romero-Rangel, S.,

y E. C. Rojas-Zenteno

ASPECTOS TAXONÓMICOS

DE LA MORFOLOGÍA

FLORAL MASCULINA DE

Quercus (FAGACEAE) EN MÉXICO

TAXONOMIC ASPECTS OF THE MALE FLORAL

MORPHOLOGY OF Quercus

(FAGACEAE) IN MEXICO

POLIBETÁNICA

Instituto Politécnico Nacional

Núm. 48: 13-27 Julio 2019

DOI:

10.18387/polibotanica.48.2

RESUMEN: La variabilidad morfológica vegetativa del subgénero Quercus ha sido objeto de un número importante de estudios para la definición de caracteres taxonómicos diagnósticos; sin embargo, la información sobre las características de las flores de las especies de México es muy escasa; por lo que este trabajo tiene como objetivo contribuir al conocimiento taxonómico de Quercus a través del estudio de la morfología floral masculina de diez especies $(Q$. crassifolia, $Q$. crassipes, $Q$. dysophylla, $Q$. frutex, $Q$. hintonii, $Q$. hypoxantha, $Q$. jonesii, $Q$. laurina, $Q$. obtusata y Q. repanda). Se estudiaron especímenes recolectados por los autores y los contenidos en los herbarios ARIZ, ENCB, IEB y MEXU. Se observaron y registraron caracteres de las inflorescencias, perianto y estambres. Se elaboraron las descripciones de las flores, se obtuvieron fotografías y se hizo un cuadro comparativo de las especies con los caracteres considerados diagnósticos. Los caracteres que aportaron información taxonómica para la delimitación de las especies fueron: largo de la inflorescencia, número de flores por inflorescencia, presencia o ausencia de pedúnculo floral, tamaño de perianto, número de estambres, tamaño de los filamentos y anteras y presencia o ausencia del apéndice apical.

Palabras clave: Fagaceae, Quercus, flores, taxonomía, México.

ABSTRACT: The vegetative morphological variability of the Quercus subgenus has been the subject of a significant number of studies for the definition of diagnostic taxonomic characters; however, information on the characteristics of the flowers of the Mexican species is very scarce; so this work aims to contribute to the taxonomic knowledge of Quercus through the study of the male floral morphology of ten species ( $Q$. crassifolia, $Q$. crassipes, $Q$. dysophylla, $Q$. frutex, $Q$. hintonii, $Q$ hypoxantha, $Q$. jonesii, $Q$. laurina, $Q$. obtusata and $Q$. repanda). Specimens collected by the authors and contained in the herbaria ARIZ, ENCB, IEB and MEXU were studied. Characteristics of the inflorescences, perianth and stamens were observed and recorded. The descriptions of the flowers were elaborated, photographs were obtained and a comparative table was made of the species with the characters considered diagnostic. The characters that provided taxonomic information for the delimitation of the species were: length of the inflorescence, number of flowers per inflorescence, presence or absence of floral peduncle, size of perianth, number of stamens, size of the filaments and anthers and presence or absence of the apical appendix.

Key words: Fagaceae, Quercus, flowers, taxonomy, Mexico. 


\section{INTRODUCCIÓN}

El género Quercus en México está representado por aproximadamente 160 especies, de las cuales más de la mitad son endémicas (Valencia, 2004). Las especies descritas en este trabajo pertenecen al subgénero Quercus y a las secciones Quercus y Lobatae (Denk, Grimm, Manos, Deng, \& Hipp, 2017). Este género comprende especies con una gran diversidad morfológica vegetativa, lo cual ha provocado se considere taxonómicamente difícil, se menciona que a esta variación ha contribuido la hibridación e introgresión (Tovar-Sánchez \& Oyama, 2004). Mientras la variabilidad morfológica vegetativa ha sido objeto de un número importante de estudios para la definición de caracteres taxonómicos diagnósticos, la información sobre las características de las flores es muy escasa, pues se considera no contribuyen al reconocimiento de los límites entre los taxa de Quercus (Romero, Rojas, \& Garay-Velázquez, 2007).

Las clasificaciones de Quercus (Denk et al., 2017; Nixon, KC, 1993) consideran caracteres florales y vegetativos para distinguir a los subgéneros y a las secciones, pero los estudios florísticos y taxonómicos de las especies de encinos mexicanos se basan primordialmente en caracteres de ramillas y hojas. Esto se puede observar en las claves de identificación y en las descripciones de las especies (Bello-González \& Labat, 1987; Romero-Rangel, Rojas-Zenteno, \& Rubio Licona, 2015; Romero Rangel, Rojas Zenteno, \& Aguilar Enríquez, 2002; Romero Rangel, Rojas Zenteno, \& Rubio Licona, 2014; Valencia, Gómez-Cárdenas, \& Becerra-Luna, 2002; Villarreal Q., Encina D., \& Carranza P., 2008).

Lo anterior ha provocado que muy pocos ejemplares de encinos de los herbarios contengan flores; además, la mayoría de ellos presentan sólo hojas inmaduras, condición que dificulta su identificación.

El género Quercus se caracteriza por tener plantas monoicas, con flores simples y unisexuales, sus flores masculinas se encuentran dispuestas en amentos colgantes provistos de un perianto 5 a 8 lobulado, con 5 a 10 estambres libres y filamentos delgados (Bello-González \& Labat, 1987; González Villarreal, 1987; Nixon, KC, 1993; Trelease, 1924; Valencia A., 2004), su polinizaciónón es anemófila. También se ha registrado la presencia de flores hermafroditas en distintas especies de México (Romero-Rangel et al., 2015; Romero Rangel, Rojas Zenteno, \& Gomez Maqueda, 2000; Romero et al., 2007).

Por lo anterior, este trabajo tiene como objetivo contribuir al conocimiento taxonómico de Quercus a través del estudio de la morfología floral masculina de diez especies $(Q$. crassifolia, $Q$. crassipes, $Q$. dysophylla, $Q$. frutex, $Q$. hintonii, $Q$. hypoxantha, $Q$. jonesii, $Q$. laurina, $Q$. obtusata y $Q$. repanda). Al mismo tiempo, intenta mostrar caracteres florales que pueden ser utilizados, junto con los vegetativos, en la definición de los límites taxonómicos de las especies de Quercus de México.

\section{MÉTODOS}

Se estudiaron especímenes recolectados por los autores en distintas localidades y los contenidos en los herbarios ARIZ, ENCB, IEB y MEXU.

Sólo se describió la morfología de flores masculinas debido a que los ejemplares de herbario con estructuras reproductoras solo contienen éstas; además en el campo, la recolección de las flores femeninas no fue posible. Tampoco se estudiaron en este trabajo las flores hermafroditas hasta ahora reportadas sólo para $Q$. glaucoides, Q. rugosa y Q. chihuahuensis (Romero-Rangel et al., 2015; Romero Rangel et al., 2000; Romero et al., 2007), pues se han observado como eventos aislados. Los autores recientemente observaron que los individuos de $Q$. rugosa que produjeron flores hermafroditas en años pasados (Romero et al., 2007) ya no las presentaron. 
Se registraron los siguientes caracteres: largo y color de inflorescencias, número de flores en las inflorescencias, pubescencia de raquis, largo de pedúnculos de flores, número de lóbulos de perianto, pubescencia, color, largo y ancho de perianto; número de estambres, largo de filamentos, largo y ancho de anteras, presencia de apéndice y tipo de base de anteras.

El número de especímenes estudiados para cada especie corresponde al total de números de colecta referidos en el apartado de material examinado.

Para la observación y medición de dichos caracteres se utilizó un microscopio Leica S6D y una retícula de $10 \mathrm{~mm} / 0.1 \mathrm{~mm}$. Las imágenes se obtuvieron con el software de Leica. Se tomaron fotografías en aumentos de 0.63 y 1.0. Con este último aumento y las flores húmedas se realizaron las mediciones de por lo menos 15 inflorescencias de cada especie; cada uno de los caracteres fue medido 15 veces, esto para cada una de las especies. La escala que se muestra en cada imagen es de $2 \mathrm{~mm}$.

Las descripciones de la morfología floral masculina se elaboraron con los datos anteriores.

Además, con la finalidad de facilitar la identificación de los taxa y la recolección futura de material reproductivo, se incluyó para cada una de las especies información reciente y actualizada como la contenida en Romero-Rangel et al. (2015), referente a los caracteres morfológicos útiles para su reconocimiento, hábitat, fenología, distribución geográfica y el riesgo de supervivencia de algunas especies según la Lista Roja de la UICN.

\section{RESUltados}

\section{Quercus crassifolia Bonpl.} Sección Lobatae

Árboles con hojas aristadas, con el envés lanoso de color amarillo, anaranjado o café y sus ramillas de 2 a $5 \mathrm{~mm}$ de diámetro. Elemento presente en los bosques de Quercus y PinusJuniperus. También se le ha encontrado en bosques perturbados de encino, en altitudes de 1300-2900 m. Florece en abril y fructifica en octubre. Especie de distribución en el occidente, centro, sur y sureste de México (Chis., Gro., Gto., Hgo., Jal., Méx., Mich., Oax., Pue., Qro., S.L.P., Tlax. y Ver.). También en Centroamérica (Guatemala).

Inflorescencias masculinas amarillentas, de (5) 7 a $9 \mathrm{~cm}$ de largo, con 35 a 45 flores, raquis con pubescencia abundante de tricomas simples; flores con pedúnculos de hasta $1 \mathrm{~mm}$ de largo, perianto café amarillento con manchas rojizas, con 4 lóbulos, pubescente, principalmente en los márgenes, de 1.5 a $1.9 \mathrm{~mm}$ de alto por 2.5 a $3 \mathrm{~mm}$ de ancho; 6 estambres, filamentos de 1.5 a $2 \mathrm{~mm}$ de largo, anteras de 1.5 a $1.9 \mathrm{~mm}$ de largo por 0.8 a $1.2 \mathrm{~mm}$ de ancho, con apéndice apical, a veces no se observa claramente, base cordada (fig.1).

Material examinado. Estado de México: Mpio. Chapa de Mota, Bioparque Estrella, RomeroRojas 9153, 9154 (IZTA); Calpulalpan, Romero-Rojas 9155 (IZTA); Mpio. Villa del Carbón, Cachihuapan, km 32 Carr. Tlalnepantla-Villa del Carbón, Romero-Rojas 9168, 9169 (IZTA). Michoacán: Mpio. Coalcomán, Sierra Torrecillas, Hinton 13697 (ARIZ).

\section{Quercus crassipes Bonpl.}

Sección Lobatae

Árboles con hojas de ápice aristado, por sus nervaduras que forman ángulos casi rectos y el envés con indumento uniforme y persistentemente pubescente. Elemento presente en los bosques de Quercus y Pinus-Quercus, a veces también en bosque mesófilo de montaña. Se le ha encontrado en sitios perturbados como claros y bordes de bosque, orillas de caminos y parcelas de maíz, en altitudes de 2000-2800 m. Florece en mayo y fructifica de septiembre a 
noviembre. Especie endémica del occidente, centro, sur y sureste de México (D.F., Gto., Hgo., Jal., Méx., Mich., Mor., Oax., Pue., Qro. y Tlax.).

Inflorescencias masculinas amarillentas a rojizas, de (3) 6 a $9 \mathrm{~cm}$ de largo, con 20 a 35 flores, raquis con pubescencia abundante de tricomas simples; flores con pedúnculos de 0.2 a $0.3 \mathrm{~mm}$ de largo, perianto café amarillento, con 4 lóbulos, pubescente, de 1.5 a $1.9 \mathrm{~mm}$ de alto por 2.8 a $3 \mathrm{~mm}$ de ancho; 6 a 7 estambres, filamentos de 1.5 a $1.7 \mathrm{~mm}$ de largo, anteras de 1.2 a 1.7 $\mathrm{mm}$ de largo por 0.7 a $1 \mathrm{~mm}$ de ancho, con apéndice apical, base cordada (fig. 2).

Material examinado. Distrito Federal: Delegación Coyoacán, extremo SW del Pedregal de San Ángel, J. Rzedowski 34696 (MEXU). Estado de México: Mpio. Jilotepec, Calpulalpan, Romero-Rojas 9159, 9160, 9161 (IZTA). Mpio. Villa del Carbón, km 34-37 Carr. TlalnepantlaVilla del Carbón, Romero-Rojas 9172 (IZTA). Jalisco: Mpio. Tolimán, 13-14 km al ENE de Minatitlán, 1-2 km al NE de El Terrero, El Tepeixtle, F. J. Santana, D. De Niz y J. Ceballos 5066 (MEXU).

\section{Quercus dysophylla Benth.}

Sección Lobatae

Árboles con hojas elípticas a lanceoladas u ovadas, con el margen entero y el ápice aristado, el envés amarillento con indumento abundante de tricomas fasciculados estipitados y la epidermis ampulosa. Elemento presente en los bosques de Quercus, Pinus-Quercus, bosque mesófilo de montaña y bosque perturbado de Quercus. Se asocia con $Q$. laurina, Q. castanea, Pinus rudis y Arbutus xalapensis, en altitudes de $2100-2800 \mathrm{~m}$. Florece de marzo a abril y fructifica de septiembre a octubre. Especie endémica del centro, sur y sureste de México (D.F., Gto., Hgo., Méx., Mich., S.L.P., Tlax. y Ver.).

Inflorescencias masculinas amarillentas, de (5) 8 a $13 \mathrm{~cm}$ de largo, con 30 a 40 flores, raquis con pubescencia abundante de tricomas simples; flores con pedúnculos de hasta $1.6 \mathrm{~mm}$ de largo, perianto muy delgado, traslúcidos, café amarillento con venas y manchas rojizas, con 4 lóbulos, casi glabro, de 1.5 a $1.8 \mathrm{~mm}$ de alto por 2.6 a $3 \mathrm{~mm}$ de ancho; 7 a 8 estambres, filamentos de 1.4 a $1.6 \mathrm{~mm}$ de largo, anteras de 1.4 a $1.9 \mathrm{~mm}$ de largo por 0.7 a $1 \mathrm{~mm}$ de ancho, sin apéndice apical (a veces se observa una pequeña proyección), base cordada y asimétrica, a veces con anteras fusionadas y otras deformadas (más pequeñas) (Fig. 3).

Material examinado. Estado de México: Mpio. Chapa de Mota, Bioparque Estrella, RomeroRojas 9156, 9157, 9158 (IZTA); Mpio. Villa del Carbón, Cachihuapan, km 32 Carr. Tlalnepantla-Villa del Carbón, Romero-Rojas 9166, 9167 (IZTA).
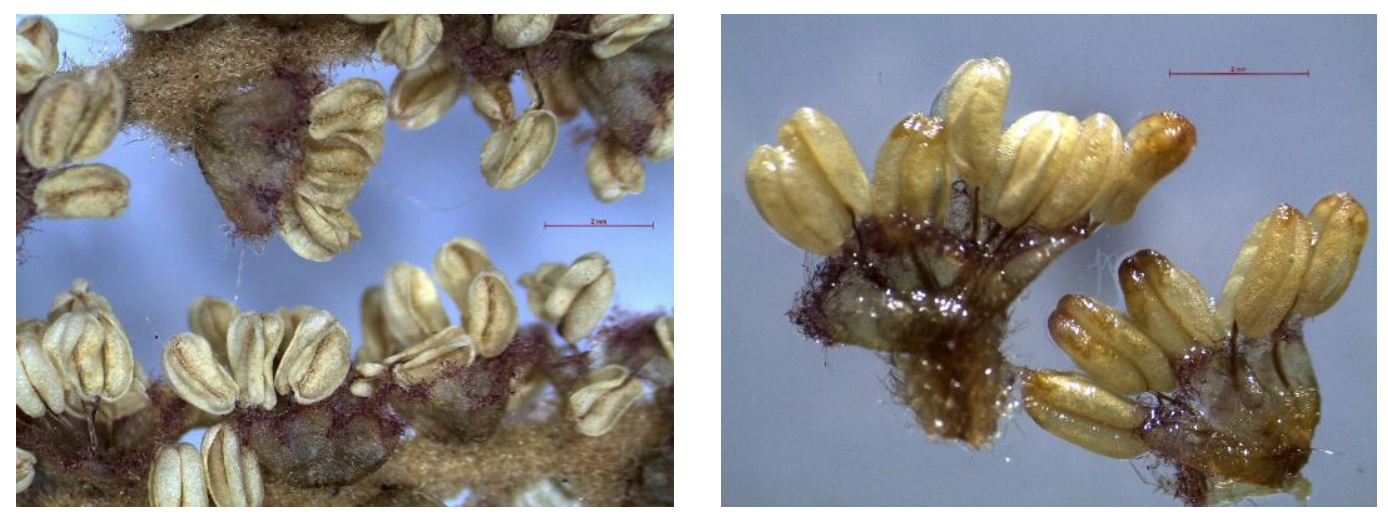

Fig. 1. Flores masculinas de Quercus crassifolia en distintos aumentos. 

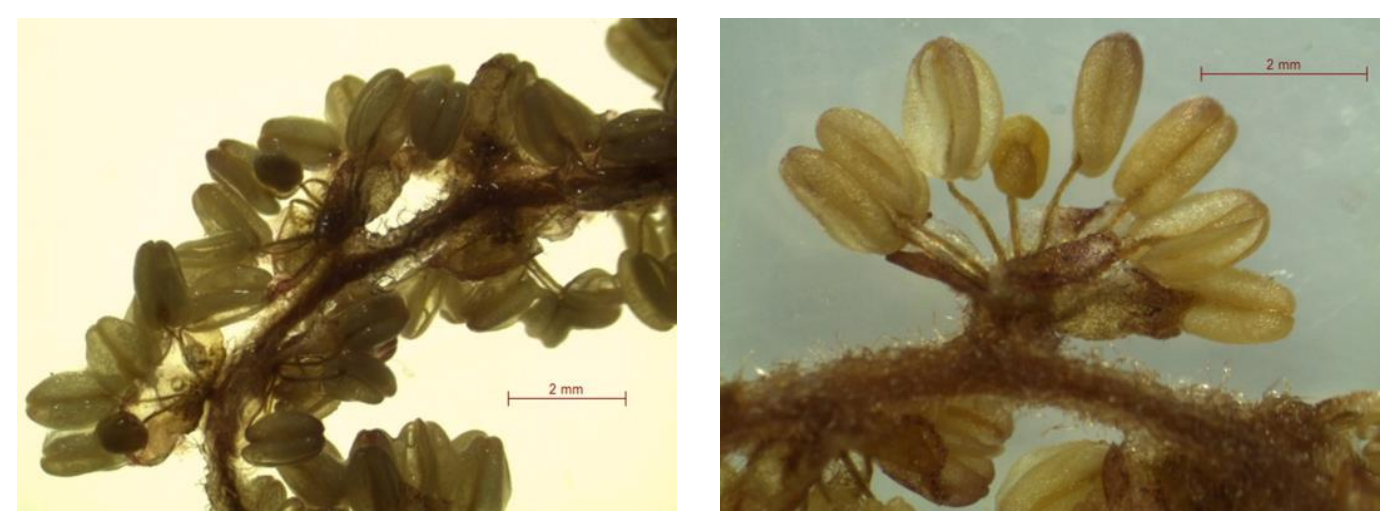

Fig. 2. Flores masculinas de Quercus crassipes en distintos aumentos.
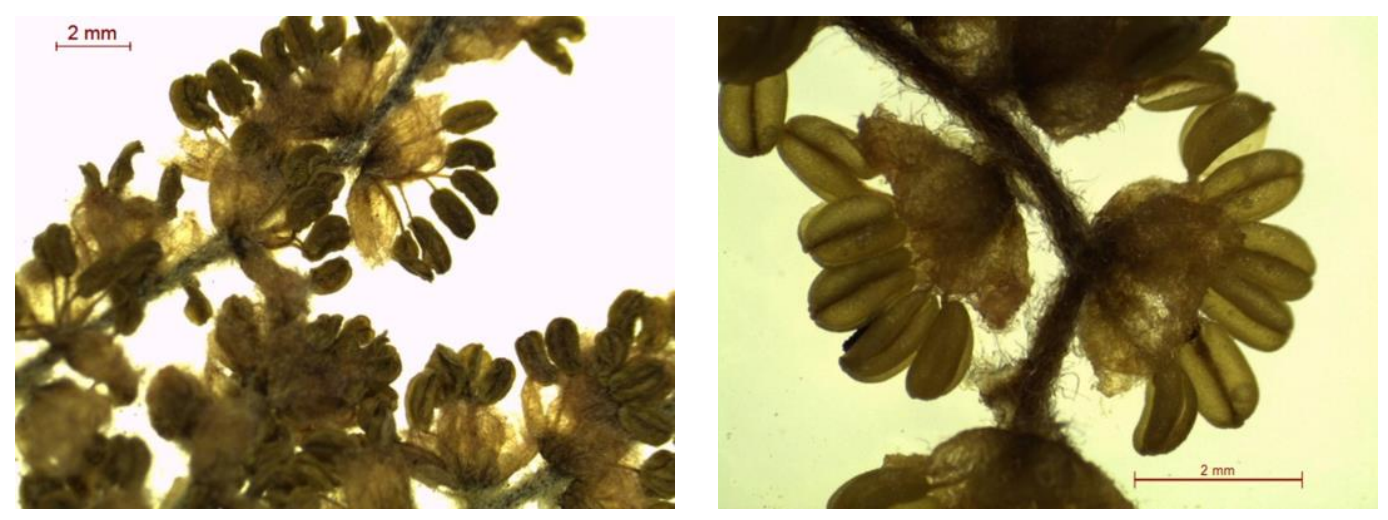

Fig. 3. Flores masculinas de Quercus dysophylla en distintos aumentos.

\section{Quercus frutex Trel.}

Sección Quercus

Arbustos rizomatosos con ramillas densamente pubescentes y hojas pequeñas, con el ápice mucronado y el margen entero, crenado o dentado-mucronado. Elemento presente en los bosques de Quercus, Pinus-Quercus y el matorral de Quercus, a veces se le encuentra en sitios perturbados con pastizal, en altitudes de 2100-3000 m. Florece en junio y fructifica de julio a septiembre. Especie endémica del occidente, centro, sur y sureste de México (D.F., Hgo., Jal., Méx., Mich., Pue., Qro., S.L.P., Tlax. y Ver.).

Inflorescencias masculinas amarillentas, de 1 a $2.5 \mathrm{~cm}$ de largo, con 15 a 20 flores, raquis con pubescencia abundante de tricomas simples; flores sésiles o con pedúnculos de hasta $0.1 \mathrm{~mm}$ de largo, perianto café amarillento con manchas rojizas, con 4 lóbulos, delgado, pubescente, de 1.5 a $1.6 \mathrm{~mm}$ de alto por 1.8 a $2.4 \mathrm{~mm}$ de ancho; 6 a 8 estambres, filamentos de 1.2 a $1.6 \mathrm{~mm}$ de largo, anteras de1.2 a $1.5 \mathrm{~mm}$ de largo por 0.9 a $1 \mathrm{~mm}$ de ancho, sin apéndice apical, base cordada (fig. 4).

Material examinado. Estado de México. Mpio. Chimalhuacán, Cerro del Pino (lado Oeste) Valle de México. J. Chavelas P. ES-1756 (NYBG); Mpio. Jilotepec, Calpulalpan, RomeroRojas 9150, 9151, 9152 (IZTA); Mpio. Villa del Carbón km 31-32 Carr. Tlalnepantla-Villa del Carbón, Romero-Rojas 9171 (IZTA). Hidalgo. Mpio. Tepeapulco, Cerca de Tepeapulco, $R$. Palacios s.n. (ENCB). Puebla. Mpio Zaragoza, San Miguel Teuxtatiloya, F. Ventura A. 21956 (MEXU). 
Quercus hintonii E. F. Warb.

Sección Lobatae

Árboles con hojas ovadas, obovadas o elípticas, aristadas, con el envés lanoso con abundante indumento que cubre totalmente la epidermis lisa. Elemento abundante a escaso en los bosques de Quercus-Pinus, se asocia con Quercus magnoliifolia, Pinus oocarpa, P. pringlei, Clethra mexicana y Juniperus flaccida, en altitudes de 1300-1950 m. Florece en marzo y fructifica de junio a agosto. Especie endémica del Estado de México. Especie con problemas de supervivencia. La Lista Roja de la UICN de Especies Amenazadas refiere a esta especie en la categoría de en peligro (EN, Endangered).

Inflorescencias masculinas amarillentas con manchas rojizas, de 5.5 a $8 \mathrm{~cm}$ de largo, con 30 a 50 flores y hasta más de 100, raquis con pubescencia abundante de tricomas simples; flores con pedúnculos de hasta $1 \mathrm{~mm}$ de largo, perianto café amarillento con manchas rojizas, con 4 lóbulos, muy pubescente, de 0.6 a $0.8 \mathrm{~mm}$ de alto por 1.8 a $2 \mathrm{~mm}$ de ancho; 5 a 7 estambres, filamentos de 1.8 a $2 \mathrm{~mm}$ de largo, anteras de 1.3 a $1.6 \mathrm{~mm}$ de largo por 0.7 a $0.8 \mathrm{~mm}$ de ancho, con apéndice apical, base cordada (fig. 5).

Material examinado. Estado de México: Mpio. Temascaltepec, Los Hornos, B. Hinton 2836 (NYBG); Tenayac, B. Hinton 5913 (NYBG); Pantoja, B. Hinton 6225 (NYBG); San Diego Cuentla, Romero-Rojas 3213, 3218 (IZTA).

\section{Quercus hypoxantha Trel.}

\section{Sección Lobatae}

Árboles con hojas coriáceas, generalmente pequeñas, con peciolos cortos, el margen dentadoserrado y fuertemente revoluto, la base cordada y el envés amarillento con el indumento denso y lanoso que se pierde con el tiempo. Elemento presente en el matorral submontano, en los chaparrales o bien en los límites de los bosques de Pinus-Quercus, bosque de Pinus o el bosque de Abies, en altitudes de $2100-2900 \mathrm{~m}$. Florece en el mes de junio y fructifica en el mes de agosto. Especie de distribución en el noreste y centro de México (Coah., N.L. y S.L.P.). Especie con problemas de supervivencia. La Lista Roja de la UICN de Especies Amenazadas refiere a esta especie en la categoría de cercanamente amenazada (NT, Near Threatened).

Inflorescencias masculinas amarillentas, de (1.5) 2 a $3 \mathrm{~cm}$ de largo, con 15 a 25 flores, raquis con pubescencia abundante de tricomas simples; flores con pedúnculos de hasta $0.3 \mathrm{~mm}$ de largo, perianto café amarillento a rojizo, con 4 lóbulos, pubescente, principalmente en los márgenes, de 1 a $1.4 \mathrm{~mm}$ de alto por 1.5 a $1.9 \mathrm{~mm}$ de ancho; 5 estambres, filamentos de 1 a $1.6 \mathrm{~mm}$ de largo, anteras de $1 \mathrm{a} 1.4 \mathrm{~mm}$ de largo por 0.6 a $0.8 \mathrm{~mm}$ de ancho, sin apéndice apical, base cordada (fig. 6).
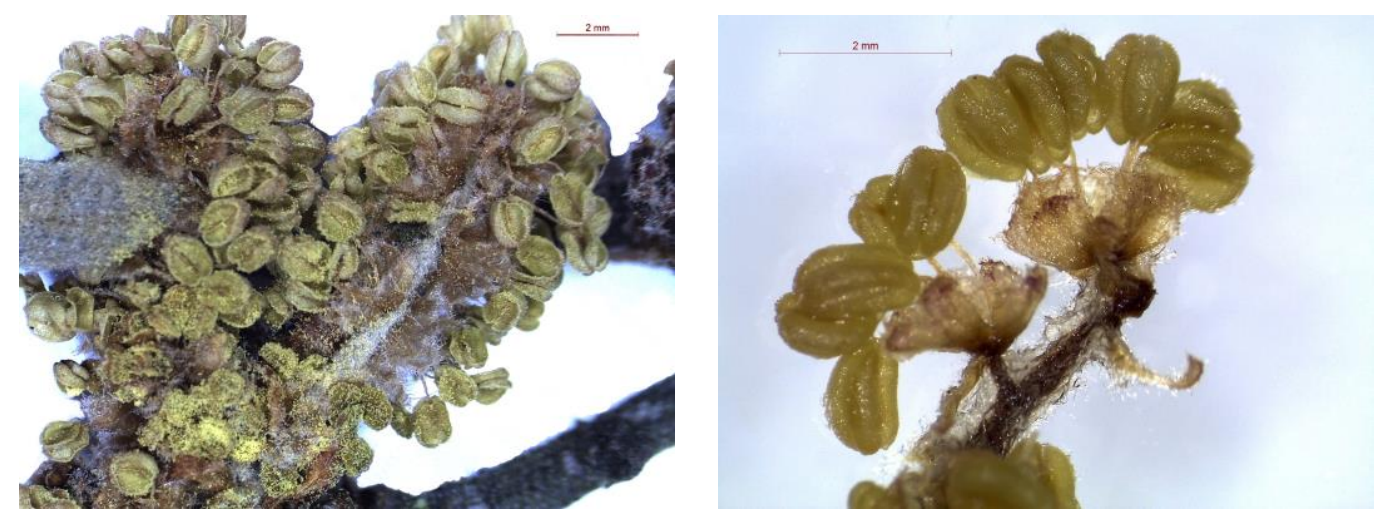

Fig. 4. Flores masculinas de Quercus frutex en distintos aumentos. 

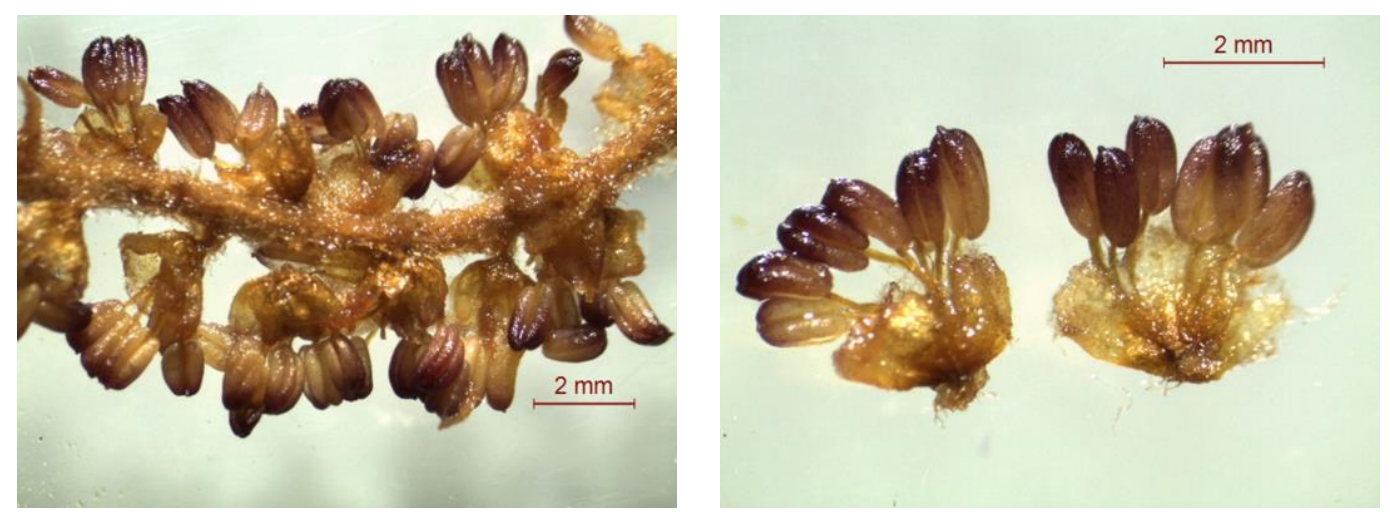

Fig. 5. Flores masculinas de Quercus hintonii en distintos aumentos.
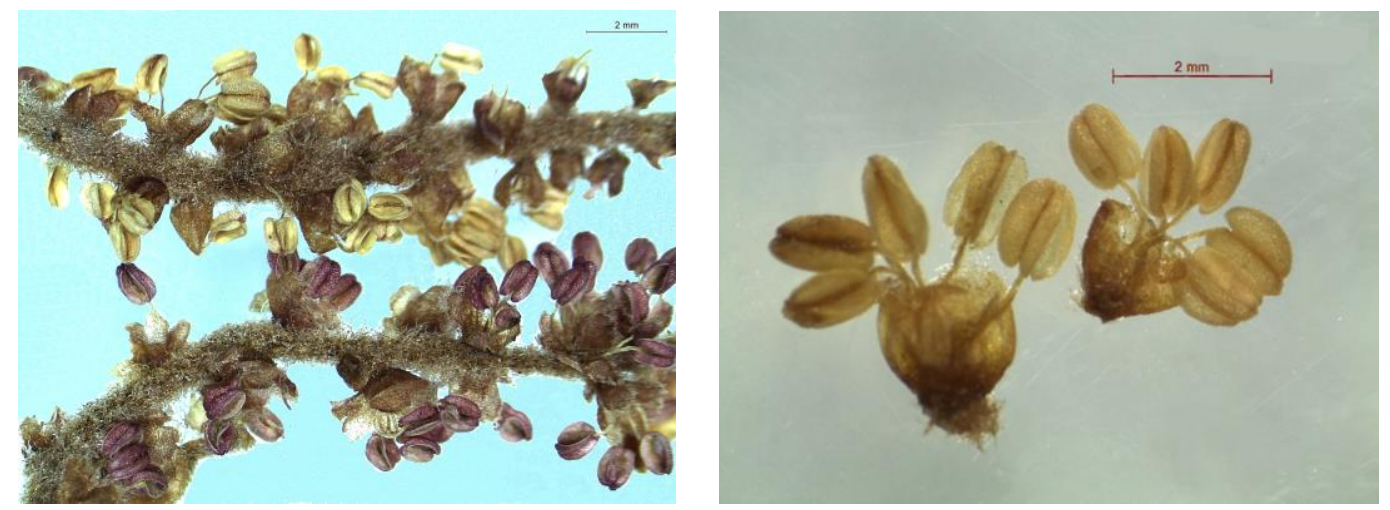

Fig. 6. Flores masculinas de Quercus hypoxantha en distintos aumentos.

Material examinado. Coahuila: Mpio. Arteaga, $4.7 \mathrm{~km}$ E of Montereal, on the way to Mesa de las Tablas. T. Yahara, M. Mishima, A. Soejima and T. Kawahara 1675 (MEXU).

Nuevo León: Mpio. Galeana. Road to summit of Cerro Potosí, ca. $7.5 \mathrm{mi}$ from Dieciocho de Marzo, L.J. Dorr 2312 (MEXU); Cerro El Potosí, B. Hinton 19457, 19460 (ENCB).

\section{Quercus jonesii Trel.}

Sección Lobatae

Árboles con hojas de textura gruesa y coriácea, ampliamente obovadas, ovadas o suborbiculares, ápice ampliamente agudo o redondeado, base cordada y con aurículas. El envés posee tricomas glandulares abundantes. Elemento presente en los bosques de Pinus-Quercus y Quercus, penetrando a sitios muy húmedos, cerca de cuerpos de agua. Se asocia con Quercus eduardii, $Q$. resinosa y $Q$. laeta, en altitudes de $1250-2450 \mathrm{~m}$. Especie endémica del noroeste, noreste, occidente y centro de México (Ags., Chih., Dgo., Gto., N.L., S.L.P. Sin., y Son.).

Inflorescencias masculinas cafés amarillentas a rojizas, de 4 a 6 (9) $\mathrm{cm}$ de largo, con 30 a 40 flores, raquis con pubescencia escasa de tricomas simples; flores con pedúnculos de hasta 0.4 $\mathrm{mm}$ de largo, perianto café amarillento a rojizo, con 4 lóbulos, pubescente sólo en los márgenes, de 1.5 a $1.8 \mathrm{~mm}$ de alto por 2.5 a $2.6 \mathrm{~mm}$ de ancho; 5 a 6 estambres, filamentos de 0.9 a $1.3 \mathrm{~mm}$ de largo, anteras de 1.8 a $2.2 \mathrm{~mm}$ de largo por 0.9 a $1.2 \mathrm{~mm}$ de ancho, sin apéndice apical, base cordada (fig. 7).

Material examinado. Guanajuato. Presa Peralvillo, Guanajuato, S. Zamudio y R. Murillo 6376 (IEB) 
Jalisco: Entre Cumbre Blanca y Mesa de los Gallos, Talpa de Allende, R. González T. 871, 872 (ENCB). Sonora. Rancho Los Alisos, near Encinal between Maycoba and El Kipor, ca $2 \mathrm{Km}$ east of Maycoba on Mex. 16, T.R. Van Devender 95-438 (ARIZ).

\section{Quercus laurina Bonpl.}

Sección Lobatae

Árboles con hojas de margen y/o ápice aristado, y el envés con indumento restringido a las axilas de las nervaduras. Elemento presente en los bosques de Quercus, en altitudes de 1600$3000 \mathrm{~m}$. Florece en abril y fructifica de octubre a diciembre. Especie de distribución en el occidente, centro, sur y sureste de México (D.F., Gro., Gto., Hgo. Jal., Méx., Mich., Mor., Oax., Pue., Qro., Tlax. y Zac.). También en Centroamérica (Guatemala y El Salvador).

Inflorescencias masculinas amarillentas, de (5) 6 a $7 \mathrm{~cm}$ de largo, con 20 a 25 flores, raquis con pubescencia escasa de tricomas simples; flores con pedúnculos de hasta $0.4 \mathrm{~mm}$ de largo, perianto café amarillento, algunos apicales con manchas rojizas, con 4 lóbulos, pubescente, de 1.5 a $2 \mathrm{~mm}$ de alto por 2.5 a $3 \mathrm{~mm}$ de ancho; 5 a 7 estambres, a veces uno más pequeño, filamentos de 1.5 a $1.9 \mathrm{~mm}$ de largo, anteras de 1.5 a $2 \mathrm{~mm}$ de largo por 0.9 a $1 \mathrm{~mm}$ de ancho, sin apéndice apical, base cordada (fig. 8).

Ejemplares Examinados. Distrito Federal: Delegación Xochimilco, Santa Cecilia, A. Ventura A. 2577 (ENCB). Estado de México: Mpio. Chapa de Mota, Parque el Llano, Romero-Rojas 9164 (IZTA). Bioparque Estrella, Romero-Rojas 9165 (IZTA).

\section{Quercus obtusata Bonpl.}

Sección Quercus

Árboles con bellotas globosas y hojas generalmente obovadas con mucrones robustos que se doblan hacia el envés, éste ligeramente ampuloso y blanco papiloso con abundantes tricomas glandulares y tricomas fasciculados con estípite corto y ramas entrelazadas dispersos en las hojas maduras. Elemento presente en los bosques de Quercus y de Pinus-Quercus, a veces penetra al bosque mesófilo de montaña, al bosque tropical caducifolio, así como a algunos pastizales y matorrales xerófilos adyacentes, en altitudes de 620-2800 m. Florece en abril y mayo, fructifica de agosto a noviembre. Especie endémica del occidente, centro, sur y sureste de México (Ags., D.F., Dgo., Gro., Gto., Hgo., Jal., Méx., Mich., Mor., Nay., Oax., Pue., Qro., S.L.P., Tlax., Ver. y Zac.).

Inflorescencias masculinas amarillentas, de 5 a $8 \mathrm{~cm}$ de largo, con 20 a 30 flores, raquis con pubescencia abundante de tricomas simples; flores sésiles, perianto café amarillento, con 4 a 5 lóbulos, pubescente, de 1.2 a $1.6 \mathrm{~mm}$ de alto por 2.2 a $2.5 \mathrm{~mm}$ de ancho; 8 (12) estambres, filamentos de 1.7 a $2.4 \mathrm{~mm}$ de largo, anteras de $1.1 \mathrm{a} 1.4 \mathrm{~mm}$ de largo por 1 a $1.2 \mathrm{~mm}$ de ancho, con apéndice apical, a veces no se observa claramente, base cordada (fig. 9).

Material examinado. Estado de México. Mpio. Villa del Carbón, San Luis Anáhuac, RomeroRojas 9101, 9166 (IZTA). Guerrero: Mpio. Mazatlán, Cerro Alquitrán, H. Kruse 2455 (MEXU). Jalisco: Mpio. Autlán, 17-18 Km al SE de Autlán, R. Cuevas y N. Núñez 3811 (MEXU). Michoacán: Mpio. Buenavista, Cerro Buenavista, predio La Alberca, F. Carrillo G. 1 (MEXU). Mpio. Charo, $15 \mathrm{Km}$ al E de Morelia, carretera Mil Cumbres, en Zurumbeneo, José Soto Núñez 6315 (MEXU). Oaxaca: Mpio. Santiago Juxtlahuaca, Barrancas de Santiago Naranjas, J. T. Calzada 21003 (IZTA). 

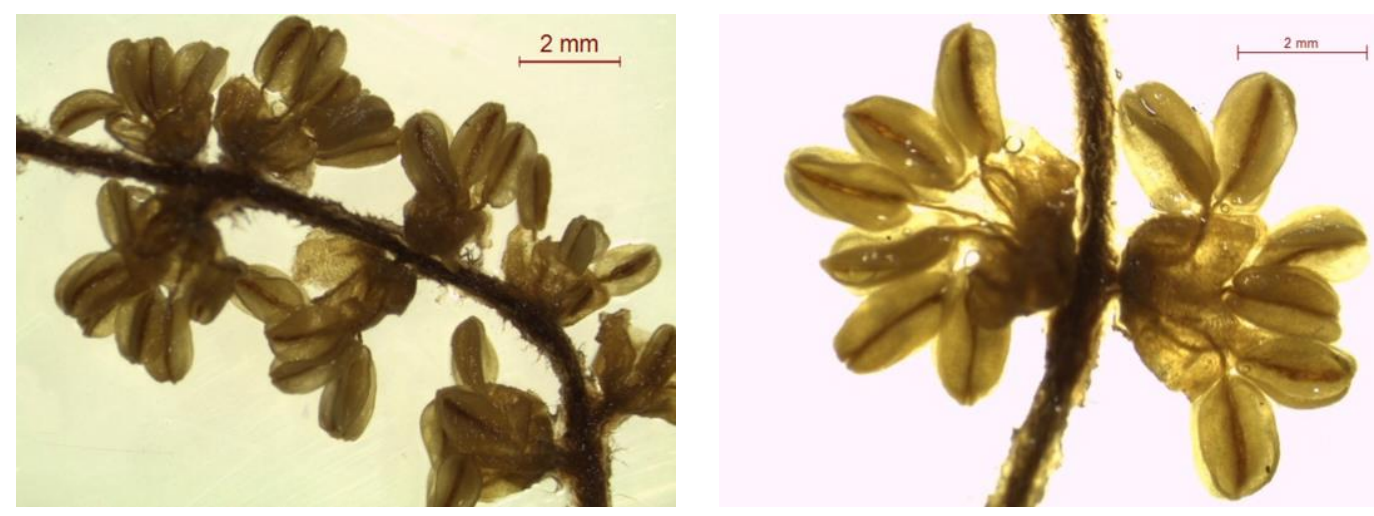

Fig. 7. Flores masculinas de Quercus jonesii en distintos aumentos.
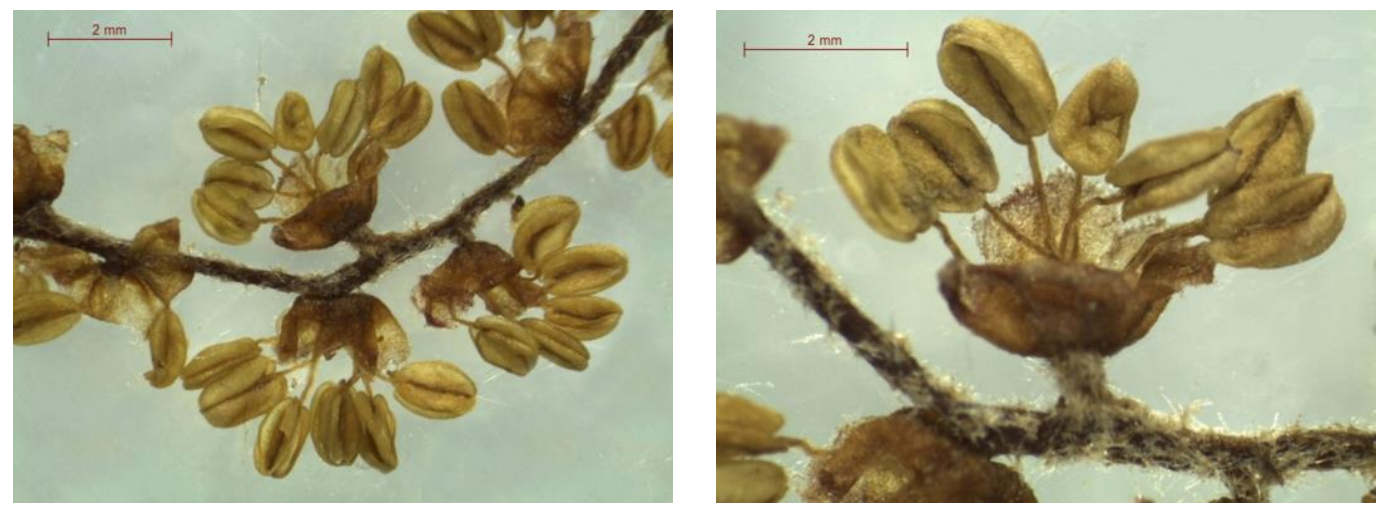

Fig. 8. Flores masculinas de Quercus laurina en distintos aumentos.
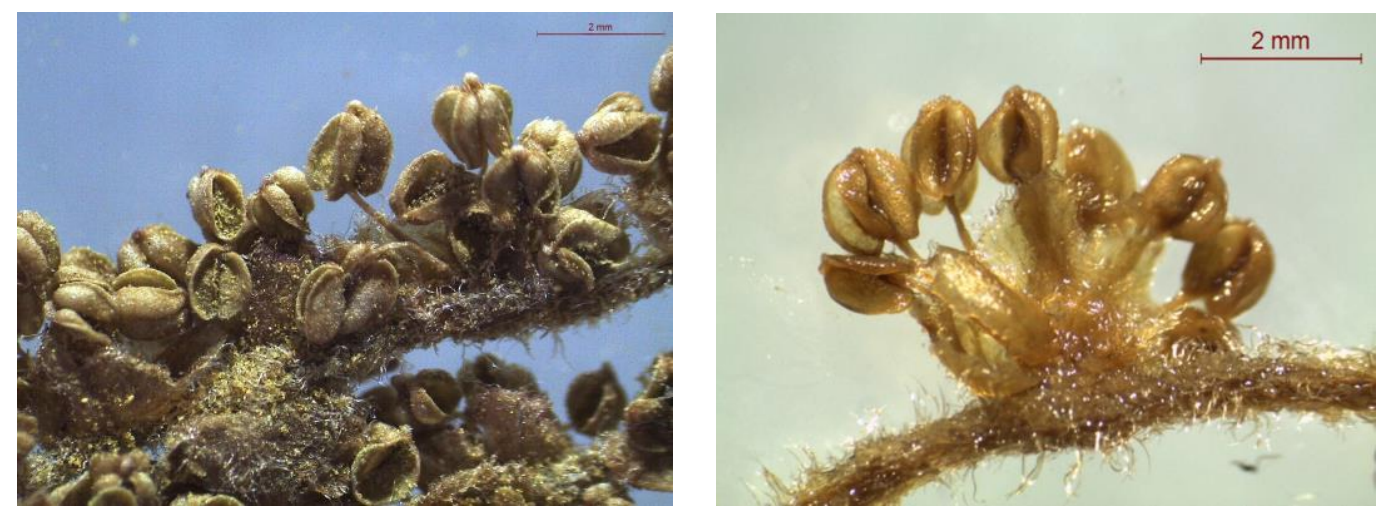

Fig. 9. Flores masculinas de Quercus obtusata en distintos aumentos.

\section{Quercus repanda Bonpl.}

Sección Quercus

Arbustos con el envés de sus hojas amarillento, con un indumento denso formado por tricomas sésiles o cortamente estipitados, que tienen 16 a 21 rayos. Elemento presente en el matorral de Quercus y en el bosque abierto de Pinus rudis mezclado con el matorral, en altitudes de 1700$3000 \mathrm{~m}$. Florece en julio y fructifica en septiembre. Especie endémica del noreste, centro, sur y 
sureste de México (Gto., Hgo., Méx., Pue., Qro., S.L.P., Tamps., Tlax., y Ver.). Especie con problemas de supervivencia. La Lista Roja de la UICN de Especies Amenazadas refiere a esta especie en la categoría de Cercanamente Amenazada (NT, Near Threatened).

Inflorescencias masculinas rojizas, de 2.5 a $4 \mathrm{~cm}$ de largo, con 20 a 30 flores, raquis con pubescencia abundante de tricomas simples; flores sésiles o con pedúnculos de $0.1 \mathrm{~mm}$ de largo, perianto café rojizo, con 4 lóbulos, pubescente, de 0.8 a $1.1 \mathrm{~mm}$ de alto por 1.4 a $1.7 \mathrm{~mm}$ de ancho; 5 a 7 estambres, filamentos de 0.9 a $1.1 \mathrm{~mm}$ de largo, anteras de 0.8 a $1.5(1.8) \mathrm{mm}$ de largo por 0.7 a $0.8 \mathrm{~mm}$ de ancho, sin apéndice apical, base cordada (fig. 10).

Material examinado. Guanajuato: Mpio. Tingambato, $2 \mathrm{~km}$ al Este de Tingambato, E. Ventura y E. López 9163 (IEB). Hidalgo: Mpio. Real del Monte, Extremo SE de Real del Monte, Miguel Medina 133 (MEXU). Puebla: Mpio. Zacatlán, Valle de Piedras Sobrepuestas, L. Wolfgang Boege 2938 (MEXU). Mpio. Zaragoza, $7 \mathrm{~km}$ al SW de Zaragoza, L. Vázquez y P. Tenorio 122 (MEXU); Querétaro: Mpio. San Joaquín, Campo Alegre, $1 \mathrm{~km}$ al SW de San Joaquín, Medina Cota y M. A. Barrios 3020 (ENCB). San Luis Potosí: Mpio. San Luis Potosí, Montes de San Miguelito, Schaffner 897 (MEXU).
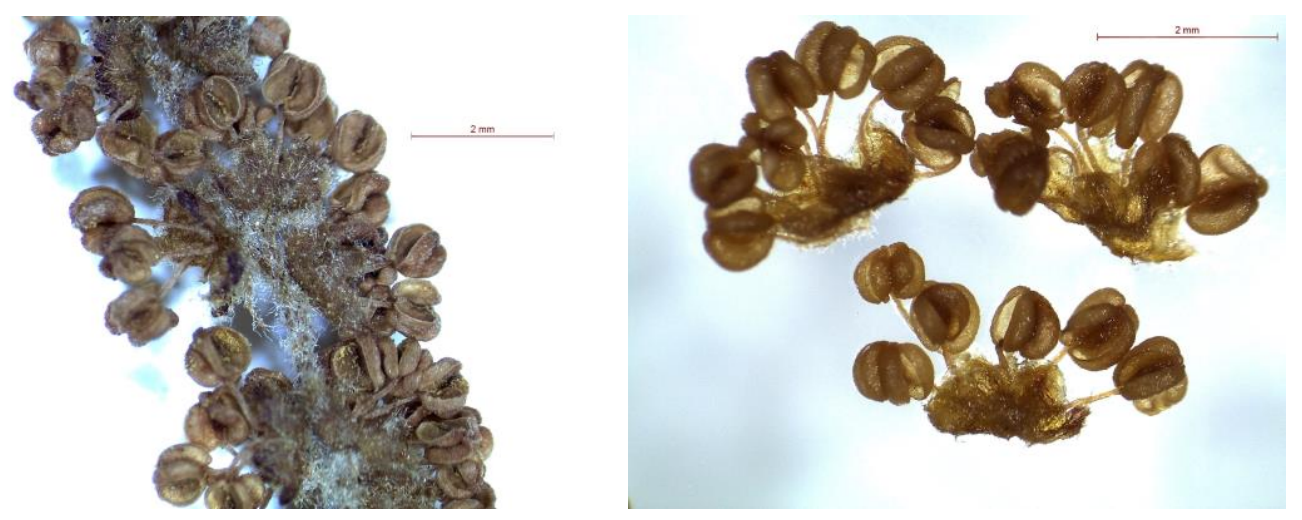

Fig. 10. Flores masculinas de Quercus repanda en distintos aumentos.

\section{DISCUSIÓN}

Este estudio no intenta la identificación de las especies sólo con base en características de las flores masculinas, sino éstas sean complementarias de las vegetativas.

Los caracteres florales, que mostraron variación entre las especies, fueron considerados diagnósticos (tabla 1); el color y la pubescencia de la inflorescencia y del perianto pueden considerarse de menor valor taxonómico, pues mostraron poca variación entre las especies. El número de lóbulos, cuatro, del perianto y el tipo de base de las anteras, cordada, se observaron sin cambio.

En siete especies se observaron inflorescencias de 5 a $13 \mathrm{~cm}$ de largo y tres las poseen de 1 a 4 cm, como son: $Q$. frutex, $Q$. hypoxantha y $Q$. repanda.

El número de flores por inflorescencia en general es de 15 a 50 , pero $Q$. hintonii puede presentar hasta más de 100; en menor cantidad se observan en $Q$. hypoxantha, $Q$. frutex y $Q$. laurina (15 a 25$)$.

Siete especies presentan flores con pedúnculos de 0.1 a $1.6 \mathrm{~mm}$, en una son sésiles, $Q$. obtusata y en dos pueden ser sésiles o con pedúnculos de $0.1 \mathrm{~mm}, Q$. frutex y $Q$. repanda. 
Tabla 1. Comparación de los caracteres diagnósticos de las diez especies en estudio.

\begin{tabular}{|c|c|c|c|c|c|c|c|c|c|c|}
\hline & Q. crassifolia & Q. crassipes & Q. dysophylla & Q. frutex & Q. hintonii & $\underset{\text { hypoxantha }}{Q .}$ & Q.jonesii & Q. laurina & Q. obtusata & Q. repanda \\
\hline $\begin{array}{l}\text { Largo de } \\
\text { inflorescencias } \\
\text { (cm) }\end{array}$ & (5) 7 a 9 & (3) 6 a 9 & (5) 8 a 13 & 1 a 2.5 & 5.5 a 8 & (1.5) 2 a 3 & 4 a $6(9)$ & (5) 6 a 7 & 5 a 8 & 2.5 a 4 \\
\hline $\begin{array}{l}\text { Núm. de flores } \\
\text { por } \\
\text { inflorescencia }\end{array}$ & 35 a 45 & 20 a 35 & 30 a 40 & 15 a 20 & $\begin{array}{c}30 \text { a } 50 \text { flores } \\
\text { y hasta más de } \\
100\end{array}$ & 15 a 25 & 30 a 40 & 20 a 25 & 20 a 30 & 20 a 30 \\
\hline $\begin{array}{l}\text { Largo de } \\
\text { pedúnculo de } \\
\text { flores }(\mathbf{m m})\end{array}$ & hasta $1 \mathrm{~mm}$ & 0.3 & hasta 1.6 & $\begin{array}{l}\text { sésiles o con } \\
\text { pedúnculos de } \\
\text { hasta } 0.1\end{array}$ & de hasta 1 & hasta 0.3 & hasta 0.4 & hasta 0.4 & sésiles & $\begin{array}{l}\text { sésiles o con } \\
\text { pedúnculos de } \\
0.1\end{array}$ \\
\hline $\begin{array}{l}\text { Alto de } \\
\text { perianto }(\mathrm{mm})\end{array}$ & 1.5 a 1.9 & 1.5 a 1.9 & 1.5 a 1.8 & 1.5 a 1.6 & 0.6 a 0.8 & $1 \mathrm{a} 1.4$ & 1.5 a 1.8 & 1.5 a 2 & 1.2 a 1.6 & 0.8 a 1.1 \\
\hline $\begin{array}{l}\text { Ancho de } \\
\text { perianto }(\mathbf{m m})\end{array}$ & 2.5 a 3 & 2.8 a 3 & 2.6 a 3 & 1.8 a 2.4 & 1.8 a 2 & 1.5 a 1.9 & 2.5 a 2.6 & 2.5 a 3 & 2.2 a 2.5 & 1.4 a 1.7 \\
\hline $\begin{array}{l}\text { Núm. de } \\
\text { estambres }\end{array}$ & 6 & 6 a 7 & $\begin{array}{l}7 \text { a } 8 \text { (anteras } \\
\text { fusionadas, } \\
\text { otras más } \\
\text { pequeñas) }\end{array}$ & 6 a 8 & 5 a 7 & 5 & 5 a 6 & $\begin{array}{c}5 \text { a } 7 \text { (a veces } \\
\text { uno más } \\
\text { pequeño) }\end{array}$ & $8(12)$ & 5 a 7 \\
\hline $\begin{array}{l}\text { Largo de } \\
\text { filamento (mm) }\end{array}$ & 1.5 a 2 & 1.5 a 1.7 & 1.4 a 1.6 & 1.2 a 1.6 & 1.8 a 2 & 1 a 1.6 & 0.9 a 1.3 & 1.5 a 1.9 & 1.7 a 2.4 & 0.9 a 1.1 \\
\hline $\begin{array}{l}\text { Largo de } \\
\text { antera }(\mathrm{mm})\end{array}$ & 1.5 a 1.9 & 1.2 a 1.7 & 1.4 a 1.9 & 1.2 a 1.5 & 1.3 a 1.6 & 1 a 1.4 & 1.8 a 2.2 & 1.5 a 2 & 1.1 a 1.4 & 0.8 a $1.5(1.8)$ \\
\hline $\begin{array}{l}\text { Ancho de } \\
\text { antera (mm) }\end{array}$ & 0.8 a 1.2 & 0.7 a 1 & 0.7 a 1 & 0.9 a 1 & 0.7 a 0.8 & 0.6 a 0.8 & 0.9 a 1.2 & 0.9 a 1 & 1 a 1.2 & 0.7 a 0.8 \\
\hline $\begin{array}{l}\text { Ápice de } \\
\text { anteras }\end{array}$ & $\begin{array}{c}\text { con apéndice } \\
\text { (a veces no } \\
\text { se observa } \\
\text { claramente) }\end{array}$ & con apéndice & sin apéndice & $\begin{array}{l}\text { sin apéndice } \\
\text { apical }\end{array}$ & $\begin{array}{c}\text { con apéndice } \\
\text { apical }\end{array}$ & $\begin{array}{l}\text { sin apéndice } \\
\text { apical }\end{array}$ & $\begin{array}{l}\text { sin apéndice } \\
\text { apical }\end{array}$ & $\begin{array}{l}\text { sin apéndice } \\
\text { apical }\end{array}$ & $\begin{array}{c}\text { con apéndice } \\
\text { apical (a veces } \\
\text { no se observa } \\
\text { claramente) }\end{array}$ & $\begin{array}{l}\text { sin apéndice } \\
\text { apical }\end{array}$ \\
\hline
\end{tabular}


El alto del perianto en siete especies va de 1.5 a $2 \mathrm{~mm}$ y es menor en $Q$. hintonii $(0.6-0.8 \mathrm{~mm})$, $Q$. hypoxantha (1-1.4 mm) y $Q$. repanda (0.8-1.1). El ancho del perianto varía de 2.4 a $3 \mathrm{~mm} ; \mathrm{y}$ es menor en $Q$. frutex (1.8-2.4), $Q$. hypoxantha (1.5-1.9) y $Q$. repanda (1.4-1.7).

El número de estambres varía de 5 a 8 , pero en $Q$. obtusata pueden observarse hasta 12 , aunque generalmente son ocho. El largo de los filamentos en ocho especies se observó de 1 a $2.4 \mathrm{~mm}$, los más pequeños se encontraron en $Q$. jonesii $(0.9-1.3 \mathrm{~mm})$ y $Q$. repanda $(0.9-1.1 \mathrm{~mm})$ y los más grandes en $Q$. obtusata $(1.7-2.4 \mathrm{~mm})$. El largo de las anteras fue de 0.8 a $2.2 \mathrm{~mm}$, en $Q$. repanda pueden observarse los más pequeños $(0.8-1.5 \mathrm{~mm})$ y los más grandes se encuentran en $Q$. jonesii $(1.8-2.2 \mathrm{~mm})$. El ancho de las anteras va de 0.6 a $1.2 \mathrm{~mm}$, los menores están en $Q$. hintonii $(0.7-0.8 \mathrm{~mm}), Q$. hypoxantha $(0.6-0.8 \mathrm{~mm})$ y $Q$. repanda $(0.7-0.8 \mathrm{~mm})$ y los más grandes en $Q$. crassifolia $(0.8-1.2 \mathrm{~mm}), Q$. jonesii $(0.9-1.2 \mathrm{~mm})$ y $Q$. obtusata $(1-1.2 \mathrm{~mm})$.

En sólo cuatro especies se presentan anteras con apéndice apical: Q. crassifolia, Q. crassipes, $Q$. hintonii y $Q$. obtusata. En algunas flores de $Q$. dysophylla se observaron anteras fusionadas y anteras de dos tamaños. También en $Q$. laurina se observó esta última condición.

Lo anterior permite observar la formación de subgrupos, pero también la presencia de caracteres exclusivos de ciertas especies.

Es conocido que los encinos muestran dificultades en la identificación de las especies, debido a su gran variación morfológica vegetativa y a la costumbre de usar sólo caracteres de ramillas, yemas y de la arquitectura foliar (forma y tamaño de hoja, nervaduras, epidermis e indumento). Además, suele incluirse la morfología del fruto, pero de modo poco preciso, debido a la escasez de ejemplares con estas estructuras.

Aunque en muchas descripciones se incluyen datos florales (como en las contenidas en Romero-Rangel et al. (2015), estos son poco precisos e incompletos y no contribuyen al reconocimiento de las especies.

Deberán describirse con mayor detalle la variación morfológica y el origen de las estructuras florales en las especies de Quercus, pues Sattler (1962) indica que en éste género las flores son tetra-pentámeras; además, Ronse De Craene (2010) menciona que en Fagaceae pueden presentarse estambres adicionales. El número de lóbulos del perianto observado en este estudio corresponde con la condición tetrámera, la cual se originó en grupos de plantas con flores pentámeras, según menciona este último autor. Es importante conocer el desarrollo de los estambres a partir de los meristemos y su posición en la flor, como lo ha demostrado Ronse De Craene (2010), a fin de que puedan considerarse como adicionales y para explicar la presencia de anteras fusionadas y de dos tamaños.

Por primera vez, para los estambres, se registra la presencia de apéndice apical en las especies en estudio y se muestra la variación del tamaño de los filamentos y anteras, pero tendrá que completarse con el análisis de una mayor cantidad de especímenes de localidades distintas.

Se recomienda la descripción detallada de la epidermis de anteras y el indumento del raquis de la inflorescencia. También, deberá describirse la morfología de las flores femeninas, en donde muy probablemente se encontrarán caracteres con valor taxonómico.

Debe estudiarse la morfología floral en relación con la polinización, pues hemos observado, durante los últimos cinco años, inflorescencias masculinas de Q. rugosa visitadas por abejas (Apis mellifera) en el Estado de México. Ronse De Craene (2010) indica que la morfología floral está directamente relacionada con la polinización y que Fagaceae está bien adaptada a la anemófila, pero algunas especies de esta familia pueden regresar a la polinización por insectos. Para incrementar el número de estudios florales será necesaria la recolección de especímenes fértiles y la observación en campo. También debe considerarse, que, aunque la floración en 
general se observa en el primer semestre del año, puede presentarse a principios del segundo, con una duración de dos a cuatro semanas.

\section{CONCLUSIONES}

Algunos caracteres de las flores masculinas descritos en este trabajo tienen valor taxonómico, los cuales pueden contribuir, junto con los caracteres vegetativos, a definir los límites taxonómicos de las especies de Quercus que habitan en México.

Los caracteres con mayor valor taxonómico fueron: largo de inflorescencia, número de flores en la inflorescencia, largo de pedúnculos de flores, largo y ancho de perianto, número de estambres, largo de filamentos, largo y ancho de anteras y presencia de apéndice en anteras. La pubescencia y el color del raquis y el perianto mostraron menos variación entre las especies. El número de lóbulos del perianto y el tipo de base de las anteras no mostraron variación.

Se sugiere incluir la morfología floral en los estudios taxonómicos y florístico-taxonómicos y realizar más trabajos en este campo.

\section{AGRADECIMIENTOS}

A Liliana E. Rubio Licona por su apoyo en la edición de la bibliografía y a los revisores por sus comentarios y sugerencias valiosas.

\section{LITERATURA CITADA}

Bello-González, M. A., \& Labat, J. N. (1987). Los encinos (Quercus) del estado de Michoacán, México. México: Secretaría de Agricultura y Recursos Hidráulicos-Centre d'Etudes Mexicaines et Centramericaines.

Denk, T., Grimm, G. W., Manos, P. S., Deng, M., \& Hipp, A. L. (2017). An Updated Infrageneric Classification of the Oaks: Review of Previous Taxonomic Schemes and Synthesis of Evolutionary Patterns. https://doi.org/10.1007/978-3-319-69099-5_2

Gonzalez Villarreal, L. M. (1987). Contribución al conocimiento del género Quercus (Fagaceae) en el estado de Jalisco.

Nixon, KC. (1993). Infrageneric classification of Quercus (Fagaceae) and typification of sectional names. Ann. For. Sci., 50, 25s-34s. https://doi.org/10.1051/forest:19930701

Romero-Rangel, S., Rojas Zenteno, C., \& Rubio Licona, L. E. (2015). Encinos de México (Quercus, Fagaceae). 100 especies. (FES-Iztacala UNAM, ed.). México: FES-Iztacala, UNAM.

Romero Rangel, S., Rojas Zenteno, C., \& Aguilar Enríquez, L. (2002). El Género Quercus (Fagaceae) en el Estado de Mexico. Annals of the Missouri Botanical Garden, (89), 551-593.

Romero Rangel, S., Rojas Zenteno, C. E., \& Gomez Maqueda, S. (2000). Flores hermafroditas de Quercus glaucoides Mart. \& Gal. (Fagaceae) en el estado de Michoacán, México. Acta Botanica Mexicana, (52), 49-54.

Romero Rangel, S., Rojas Zenteno, C., \& Rubio Licona, L. E. (2014). Fagaceae. Flora del Bajio y de Regiones Adyacentes.

Romero, S., Rojas, E. C., \& Garay-Velázquez, O. H. (2007). Presencia de flores hermafroditas en Quercus rugosa (Fagaceae) en el Estado de México (México). In Anales del Jardín Botánico de Madrid (Vol. 64). 
Recibido:

18/diciembre/2018

Aceptado:

8/junio/2019
Ronse De Craene, L. (2010). Floral diagrams: An aid to understanding flower morphology and evolution. In Floral Diagrams: An Aid to Understanding Flower Morphology and Evolution. https://doi.org/10.1017/CBO9780511806711

Sattler, R. (1962). Zur fruÈhen Inoreszenz-und BluĖtenentwicklung der Primulales sensu lato mit besonderer BeruÈcksichtigung der StamenĐPetalum-Entwicklung. Botanische JahrbuÈcher FuĖr Systematik, Panzengeschichte Und Panzengeographie, (81), 358396.

Tovar-Sánchez, E., \& Oyama, K. (2004). Natural hybridization and hybrid zones between Quercus crassifolia and Quercus crassipes (Fagaceae) in Mexico: Morphological and molecular evidence. In American journal of botany (Vol. 91). https://doi.org/10.3732/ajb.91.9.1352

Trelease, W. (1924). The American oaks /. Retrieved from https://www.biodiversitylibrary.org/item/242051

Valencia A., S. (2004). Diversidad del género Quercus (Fagaceae) en México. Boletín de la Sociedad Botánica de México, (75), 33-53. Retrieved from https://www.redalyc.org/articulo.oa?id=57707503

Valencia, S., Gómez-Cárdenas, M., \& Becerra-Luna, F. (2002). Catálogo de encinos del estado de Guerrero, México. Libro Técnico, (1).

Villarreal Q., J. A., Encina D., J. A., \& Carranza P., M. A. (2008). Los Encinos (Quercus: Fagaceae) de Coahuila, México. Journal of the Botanical Research Institute of Texas, 2(2), 1235-1278. Retrieved from http://www.jstor.org/stable/41971768 